\title{
Antiarrhythmic medication induced multi-organ dysfunction
}

\begin{abstract}
Propafenone is a Class $1 \mathrm{C}$ drug indicated for reduction of episodes of paroxysmal atrial fibrillation. There is a paucity of literature to guide the escalation of propafenone in a patient who has been on long-term therapy. Multi-organ toxicity following dose escalation within the recommended therapeutic dose range appears to be a rare event. We report a patient who presented with severe systolic heart failure six weeks after propafenone dose escalation. We discontinued propafenone therapy and the patient had full clinical and echocardiographic recovery
\end{abstract}

Keywords: propafenone, cardiotoxicity, hepatotoxicity, dose escalation
Volume 6 Issue I - 2017

Enoch Lule,' OlisaAchike, ' Yashwant Agrawal, ${ }^{2}$ Jagadeesh K Kalavakunta ${ }^{1,2,3}$

'Department of Internal Medicine/Cardiology, Michigan State University, USA

${ }^{2}$ Department of Internal Medicine \&Pediatrics, Western Michigan University Homer Stryker School of Medicine, USA

${ }^{3}$ Department of Cardiology, Borgess Medical Center, USA

Correspondence: Jagadeesh K Kalavakunta, Department of Internal Medicine/Cardiology, Borgess Medical Center, Kalamazoo, Michigan, USA, Email jkalavakunta@gmail.com

Received: January 08, 2017| Published: February 01, 2017
Abbreviations: AF, atrial fibrillation; ECG, electrocardiogram; TTE, transthoracic echocardiogram; INR, international normalized ratio; SVT, supraventricular tachycardia

\section{Introduction}

Propafenone used in the treatment of atrial fibrillation and supraventricular tachycardia is a class IC anti-arrhythmic medication which is generally safe at when administered in the thera peutic range. Under rare circumstances, toxicity involving the cardiovascular system can occur.

\section{Case report}

A 77-year-old Caucasian female with history of chronic atrial fibrillation (AF) on propafenone $150 \mathrm{mg}$ three times daily was admitted for uncontrolled ventricular rates and dyspnea on exertion. Physical exam was relevant for irregularly irregular heart rate at 145 beats per minute (bpm). Her initial electrocardiogram (ECG) revealed atrial fibrillation at a rate of 145 beats per minute (Figure 1). Labs and transthoracic echocardiogram (TTE) were in normal limits. Her propafenone was increased to $225 \mathrm{mg}$ three times daily which did not improve her AF. She was then successfully electrically cardioverted to normal sinus rhythm two days later and was discharged on this increased dose of propafenone.

She was admitted 6weeks later with dyspnea and palpitations. Physical exam revealed irregularly irregular heart rate. Labs showed elevated liver enzymes (AST- 1049U/L, ALT-577U/L) and international normalized ratio (INR) of 23. Labs for viral hepatitis and autoimmune hepatitis were negative. ECG revealed AF with heart rate of $111 \mathrm{bpm}$ with left bundle branch block (Figure 2). TTE was significant for ejection fraction of $20 \%$ and paradoxical septal wall motion. Propafenone toxicity was high on the differential was causing the present clinical picture and it was discontinued along with warfarin which she was on for anticoagulation.

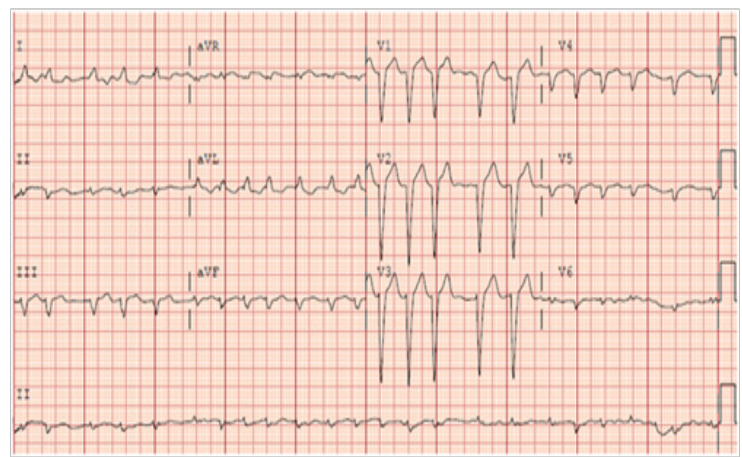

Figure I EKG at initial presentation showing atrial fibrillation at a rate of I45bpm.

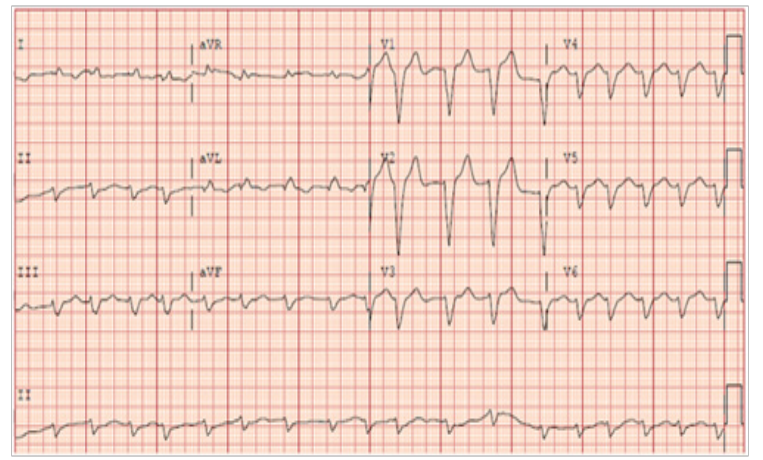

Figure 2 EKG upon readmission 6weeks later. It revealed atrial fibrillation with a heart rate of I I Ibpm with left bundle branch block.

After discontinuation of the propafenone, she improved clinically with normalization of transaminitis, INR, ECG showing rate of $88 \mathrm{bpm}$ with loss of left bundle branch block and TTE showing 60$65 \%$ ejection fraction on day 10 of her admission. She was discharged and has been doing well at her outpatient follow up appointments. 


\section{Discussion}

Propafenone (2'-[3-(propyl amino)-2-(hydroxy)-propoxy]-3phenylpropiophenone hydrochloride) is a Vaughn Williams Class 1C antiarrhythmic drug with proven efficacy in reduction of episodes of supraventricular tachycardia (SVT) and AF. ${ }^{1,2}$ Guidelines on AF management state that propafenone may be initiated as an out-patient if care is taken to ensure the patient has no structural heart disease, no sinus node or atrio-ventricular node disease and no Brugada pattern on $\mathrm{EKG}^{3}{ }^{3}$

Propafenone increased the median free interval without recurrence of arrhythmia to 44days in the ERAFT (European Rythmol/Rytmonorm Atrial Fibrillation Trial) trial, ${ }^{1}$ a mean of 98days in the PSVT study ${ }^{2}$ and over 300days in the RAFT (Rythmol Atrial Fibrillation Trial) trial. ${ }^{4}$ Propafenone has also been shown to be effective in converting patients with recent onset of atrial fibrillation with reported success rates of conversion between 72 and $94 \%{ }^{5,6} \mathrm{~A}$ recent meta- analysis found that unlike flecainide, propafenone does not have an adverse effect on mortality and it is not pro-arrhythmic. ${ }^{7}$

In the study of patients with PSVT or atrial fibrillation, congestive heart failure occurred in $1.9 \%$ of patients. ${ }^{2}$ Reduction in ejection fraction is confined to those with preexisting reduced ejection fraction. ${ }^{8}$ Liver toxicity is a rare side effect of propafenone. In a review of nine cases reported in literature propafenone toxicity was noted a median of 4weeks after propafenone initiation. ${ }^{9}$ A nonlinear relationship between dose and steady state concentrations has been noted. This means a large increase in plasma concentration can result from a small dose increase. ${ }^{10} \mathrm{We}$ did not come across recommendations when adjusting the dose of propafenone on patients who have been on chronic therapy. We did however find two other cases in literature of marked reduction in systolic function in patients who had been on long term therapy with propafenone and had undergone a medication dose increase. ${ }^{11,12}$

Our patient is noteworthy for a few reasons. Her ejection fraction fell from normal to an estimated $20 \%$. From our review of literature, it appears such a precipitous decline in ejection fraction with propafenone dose increases in the usual therapeutic range is rare. The most remarkable finding in her ECG at the time of maximal toxicity (when her QRS interval was 176) is the marked left bundle branch block. This caused marked dyssynchrony noted on her TTE.

We believe that our patient's cardiac and hepatic dysfunction was caused by propafenone considering the sub-acute worsening of her clinical picture after propafenone dose increase, and subsequent improvement after discontinuation of the medication.

\section{Conclusion}

Propafenone is an effective drug in converting patients with recent onset $\mathrm{AF}$ and reducing recurrences of $\mathrm{AF}$. Although rare, propafenone toxicity should be considered in patients having symptoms of systolic heart failure and hepatotoxicity soon after a dose increase. Monitoring for three days post dose increase may be insufficient in judging the risk of toxicity. It may be reasonable to review ECGs prior to any exposure to propafenone when making dose adjustments much later.

\section{Acknowledgements}

None.

\section{Conflict of interest}

The author declares no conflict of interest.

\section{References}

1. Meinertz T, Lip GY, Lombardi F, et al. Efficacy and safety of propafenone sustained release in the prophylaxis of symptomatic paroxysmal atrial fibrillation (The European Rythmol/Rytmonorm Atrial Fibrillation Trial [ERAFT] Study). Am J Cardiol. 2002;90(12):1300-1306.

2. Cobbe SM, Rae AP, Poloniecki JD, et al. A randomized, placebocontrolled trial of propafenone in the prophylaxis of paroxysmal supraventricular tachycardia and paroxysmal atrial fibrillation. UK Propafenone PSVT Study Group. Circulation. 1995;92(9): 2550-2557.

3. Fuster V, Rydén LE, Asinger RW, et al. ACC/AHA/ESC guidelines for the management of patients with atrial fibrillation: executive summary: a report of the American College of Cardiology/American Heart Association Task Force on Practice Guidelines and the European Society of Cardiology Committee for Practice Guidelines and Policy Conferences (Committee to Develop Guidelines for the Management of Patients With Atrial Fibrillation). Circulation. 2001;104: 2118-2150.

4. Pritchett E, Page RL, Carlson M, et al. Efficacy and safety of sustainedrelease propafenone (propafenone SR) for patients with atrial fibrillation. Am J Cardiol. 2003;92(8): 941-946.

5. Boriani G, Martignani C, Biffi M, et al. Oral loading with propafenone for conversion of recent onset atrial fibrillation: a review on in-hospital treatment. Drugs. 2002;62(3): 415-423.

6. Alboni P, Botto G, Baldi N, et al. Outpatient treatment of recent-onset atrial fibrillation with the "pill-in-the-pocket" approach. $N$ Engl J Med. 2004;351(23): 2384-2391.

7. Lafuente-Lafuente C, Longas-Tejero MA, Bergmann JF, et al. Antiarrhythmics for maintaining sinus rhythm after cardioversion of atrial fibrillation. Cochrane Database Syst Rev. 2012;5:CD005049.

8. Shen EN, Sung RJ, Morady F, et al. Electrophysiologic and hemodynamic effects of intravenous propafenone in patients with recurrent ventricular tachycardia. J Am Coll Cardiol. 1984;3(5): 1291-1297.

9. Younan LB, Barada KA, Faraj WG, et al. Propafenone hepatotoxicity: report of a new case and review of the literature. Saudi J Gastroenterol. 2013;19(5): 235-237.

10. Connolly SJ, Kates RE, Lebsack CS, et al. Clinical pharmacology of propafenone. Circulation. 1983;68(3):589-596.

11. Wylie J, Zimetbaum P, Josephson M, et al. Cardiac memory induced by QRS widening due to propafenone toxicity. Pacing Clin Electrophysiol. 2007;30(9):1161-1164.

12. Samaan RA, Sobamowo HO, Tamburrino F, et al. Syncope, widened QRS interval, and left ventricular systolic depression: coincident with propafenone therapy for atrial fibrillation. Tex Heart Inst J. 2010;37(4):476-479. 